\title{
AVALIAÇÃO BIOMECÂNICA DA FIXAÇÃO DA FRATURA SUPRACONDILIANA DO FÊMUR COMPARANDO PLACA-LÂMINA 95 COM DCS
}

\author{
FIXATION OF SUPRACONDYLAR FEMORAL FRACTURES: A BIOMECHANICAL ANALYSIS \\ COMPARING THE 95० BLADE PLATE AND THE DYNAMIC CONDYLAR SCREW (DCS)
}

Marco Antônio Percope Andrade', André Soares Rodrigues², Celso Junio Mendonça ${ }^{3}$, Luiz Gustavo Santos Portela ${ }^{3}$

\section{RESUMO}

Objetivo: Demonstrar por meio de ensaios biomecânicos comparativos entre a placa-lâmina de $95^{\circ}$ e o parafuso condilar dinâmico (Dynamic Condylar Screw - DCS), qual apresenta maior resistência às cargas compressivas e de flexão, bem como tentar correlacionar o tipo de falha apresentada durante os testes com cada um dos tipos de placa. Métodos: Sessenta e cinco fêmures suínos foram submetidos a osteotomia em cunha de subtração medial de um centímetro $(\mathrm{cm})$, na região metafisária distal do fêmur, com o objetivo de simular fratura supracondiliana instável. Foi realizada osteossíntese dessas peças, sendo 35 fixadas com placa-lâmina 95 e 30 com placas com DCS, submetendo-as a cargas em compressão axial e flexão. Outra variável estudada foi o tipo de falha apresentada em cada grupo com a tentativa de correlacioná-la com o tipo de placa. Resultados: Os resultados não mostraram diferença estatisticamente significante na resistência biomecânica entre os dois tipos de placas ou entre o tipo de falha e a placa utilizada na osteossíntese. Conclusão: Os dois tipos de placas se comportam de maneira semelhante, embora haja um indicativo de que a placalâmina seja, no ensaio de flexão, superior à placa DCS. Não foi observada diferença entre o tipo de falha e o tipo de placa utilizada.

Descritores - Fraturas do fêmur; Fixação interna de fraturas; Biomecânica

\section{ABSTRACT}

Objective: to determine, by means of comparative biomechanical tests between the $95^{\circ}$ angled blade plate and the dynamic condylar screw (DCS), the one that presents greater compressive load resistance and flexion, and to correlate the failure type presented during the tests with each type of plate. Methods: Sixty-five porcine femurs were submitted to a $1 \mathrm{~cm}$ medial wedge osteotomy, in the metaphysic, to simulate an unstable supracondylar femoral fracture. Osteosynthesis of these pieces was performed: 35 were fixated with a $90^{\circ}$ lateral blade plate, and 30 with DCS plates. Another variable studied was the failure type presented in each group, attempting to correlate this with the type of plate. Results: The results showed no statistically significant differences in biomechanical resistance between the two types of plates, or between the failure type and the plate type used in the osteosynthesis. Conclusion: The two types of plate behaved in a similar fashion. However, the angled blade plate proved to be superior to the DCS in the flexion test. No statistical difference was observed in failure type or type of plate used.

Keywords - Femoral fractures; Fracture fixation, internal; Biomechanics

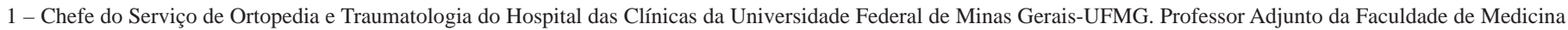
da UFMG.

2 - Ortopedista, especialista em Cirurgia do Quadril. Membro do Corpo Clinico do Hospital Universitário Risoleta Tolentino Neves (UFMG).

3 - Ex-Residente do Hospital das Clínicas da Universidade Federal de Minas Gerais-UFMG.

Trabalho desenvolvido no Hospital das Clínicas e no Campus da Universidade Federal de Minas Gerais.

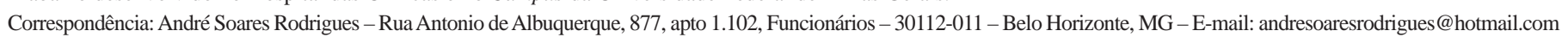

Declaramos inexistência de conflito de interesses neste artigo 


\section{INTRODUÇÃO}

As fraturas da região supracondiliana do fêmur são lesões complexas, habitualmente de difícil tratamento. Correspondem a aproximadamente $7 \%$ de todas as fraturas do fêmur e a 31\% delas, se excluídas as fraturas do fêmur proximal ${ }^{(1)}$. A causa mais frequente na população idosa é a queda da própria altura com o joelho fletido e, em pacientes jovens, é o trauma de alta energia (acidentes automobilísticos, quedas de altura), geralmente levando a forças em varo, valgo ou rotacionais com carga axial. As lesões ligamentares podem estar associadas em torno de $20 \%$ dos $\operatorname{casos}^{(1)}$, assim como fraturas do acetábulo, do colo e diáfise femorais e do platô tibial.

Uma característica peculiar dessa fratura é a deformidade causada pela ação dos diversos grupos musculares agindo no joelho (quadríceps, isquiotibiais, gastrocnêmio e adutores), levando a desvios dos fragmentos, principalmente em hiperextensão pela ação do gastrocnêmio, havendo necessidade de redução cruenta e fixação interna.

O melhor método de fixação das fraturas supracondilianas do fêmur ainda é motivo de controvérsia. Vários implantes são utilizados para a fixação desse tipo de fratura sem, no entanto, haver consenso a respeito de qual seria o mais estável biomecanicamente. Além da fixação intramedular retrógrada, destacam-se as placas com lâmina angulada a $95^{\circ}$ e as placas com parafuso condilar dinâmico DCS (Dynamic Condylar Screw).

A placa-lâmina proporciona excelente fixação e é considerada a de maior resistência às forças de angulação e torção, apesar da maior dificuldade técnica ${ }^{(2-3)}$. Já o DCS, pelo fato de ser mais espesso que a placalâmina, provoca, em teoria, maior destruição óssea ${ }^{(2)}$. Estudos comparando o DCS com hastes intramedulares retrógradas e anterógradas mostram resultados conflitantes ${ }^{(4-7)}$.

O propósito deste estudo é comparar a rigidez biomecânica da fixação interna realizada com placalâmina $95^{\circ}$ e com o DCS, em simulação de fraturas supracondilianas extra-articulares instáveis em fêmur de suínos, bem como correlacionar os tipos de placa com o tipo de falha, se óssea (fratura) ou se do material utilizado (soltura ou quebra do implante). Esses implantes foram escolhidos por ser os mais comumente utilizados no tratamento do tipo de fratura estudado em nosso meio.

\section{MÉTODOS}

Sessenta e cinco fêmures suínos da raça Landraz, com idade média de 90 dias, foram selecionados para o experimento. Os ossos foram armazenados à temperatura de $-18^{\circ} \mathrm{C}$ e então colocados em temperatura ambiente 12 horas antes de ser fixados.

Foram utilizadas na fixação dos fêmures 65 placas, todas com cinco orifícios, sendo 35 com lâmina angulada a $95^{\circ}$ de 55 milímetros (mm) e 30 com parafuso condilar dinâmico (DCS) de 55mm de comprimento, fixando-se o furo mais distal de ambas as placas com um parafuso esponjoso e três parafusos corticais nos furos proximais, deixando-se sem fixação o furo correspondente à região da osteotomia, que foi realizada a cerca de $4 \mathrm{~cm}$ da superfície articular. Para osteossíntese foram utilizados os princípios e técnicas do grupo $\mathrm{AO} /$ $\mathrm{ASIF}^{(8)} \mathrm{com}$ placas e parafusos produzidos pela empresa I.O.L. Implantes Ltda.

Após a fixação dos fêmures foi realizada uma osteotomia em cunha de subtração medial de $1 \mathrm{~cm}$ na região metafisária distal do fêmur, utilizando-se serra oscilatória de nitrogênio, com a finalidade de se criar uma fratura supracondiliana instável (sem suporte medial) (Figura 1).

Os ossos após a fixação foram novamente armazenados à temperatura de aproximadamente $-18^{\circ} \mathrm{C}$ e, 20 horas antes do início do experimento, transferidos para um refrigerador com temperatura de cerca de $4^{\circ} \mathrm{C}$, sendo então, uma hora antes dos testes, deixados à temperatura ambiente.
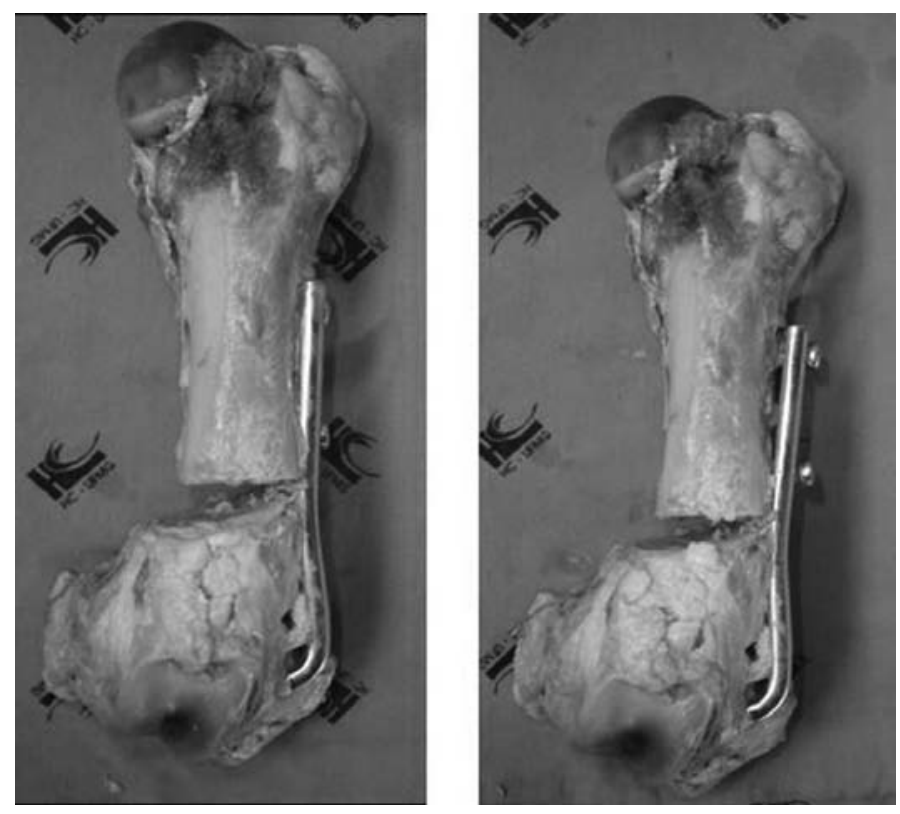

Figura 1 - Peça fixada e submetida à osteotomia em cunha de subtração medial, antes e após o teste de flexão 
Para a realização do ensaio biomecânico, foram utilizadas as instalações do laboratório de ensaios mecânicos do Centro de Desenvolvimento de Tecnologia Nuclear da Comissão Nacional de Energia Nuclear (CDTN/ CNEN), sendo as peças submetidas a cargas de compressão axial e flexão na máquina de ensaios universal Instron TTDML ${ }^{\circledR}$ (Canton, MA, EUA), com capacidade máxima de 10 toneladas (Figura 2).

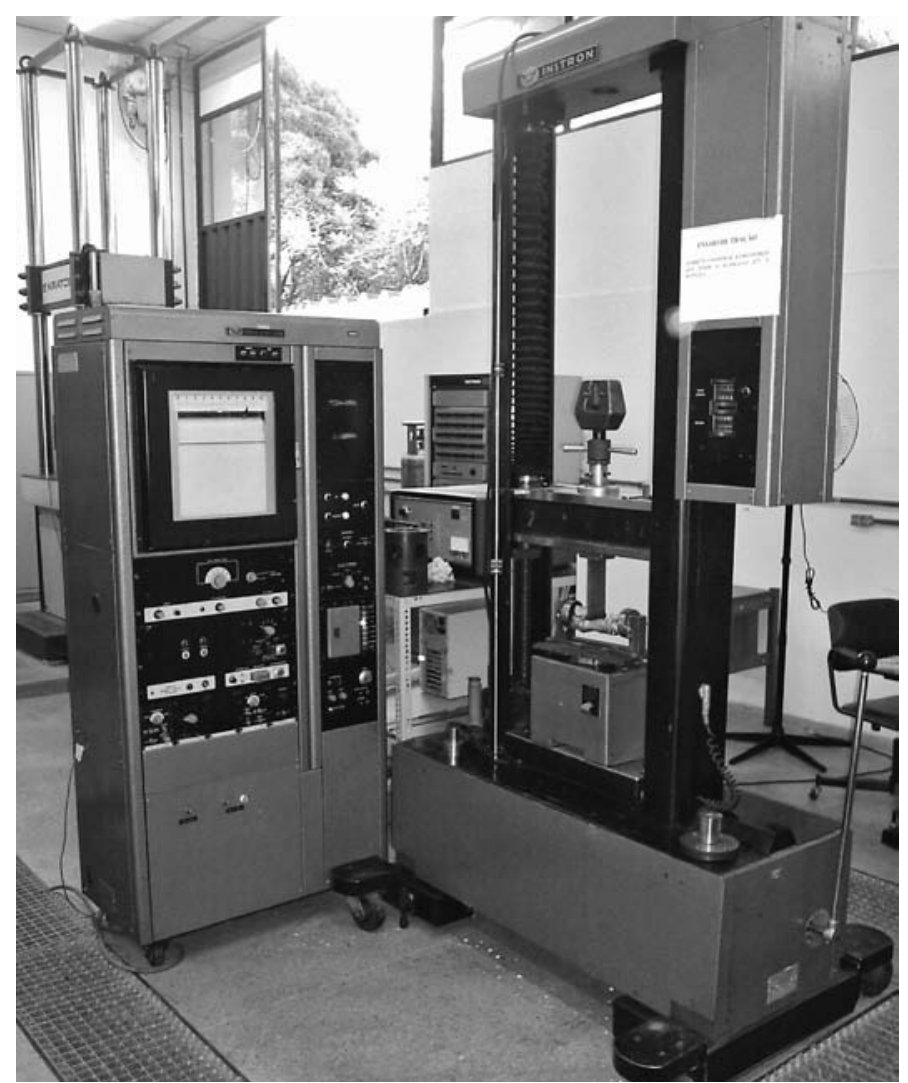

Figura 2 - Máquina de ensaios universal Instron TTDML ${ }^{\circledR}$ (Canton, MA, EUA. Laboratório de ensaios mecânicos do Centro de Desenvolvimento de Tecnologia Nuclear da Comissão Nacional de Energia Nuclear (CDTN/CNEN)

Suportes metálicos foram confeccionados para a perfeita adaptação das peças à máquina de ensaios durante a aplicação das cargas, no intuito de se evitar qualquer tipo de movimentação que pudesse falsear o ponto de falha mecânica. No teste de compressão não se permitiu o contato dos suportes com o material de síntese para evitar que as cargas aplicadas ao conjunto placa-osso fossem transmitidas de forma inadequada. No teste de flexão buscou-se aplicar a carga no terço médio da diáfise, na cortical diametralmente oposta à do material de síntese (Figura 3).

As peças foram randomizadas em quatro grupos de acordo com o tipo de fixação e com o tipo de teste utilizado. Assim, 20 peças fixadas com placa-lâmina e 15 com DCS foram submetidas a cargas em flexão, e 15 fixadas com placa-lâmina e 15 com DCS foram submetidas a cargas em compressão axial.
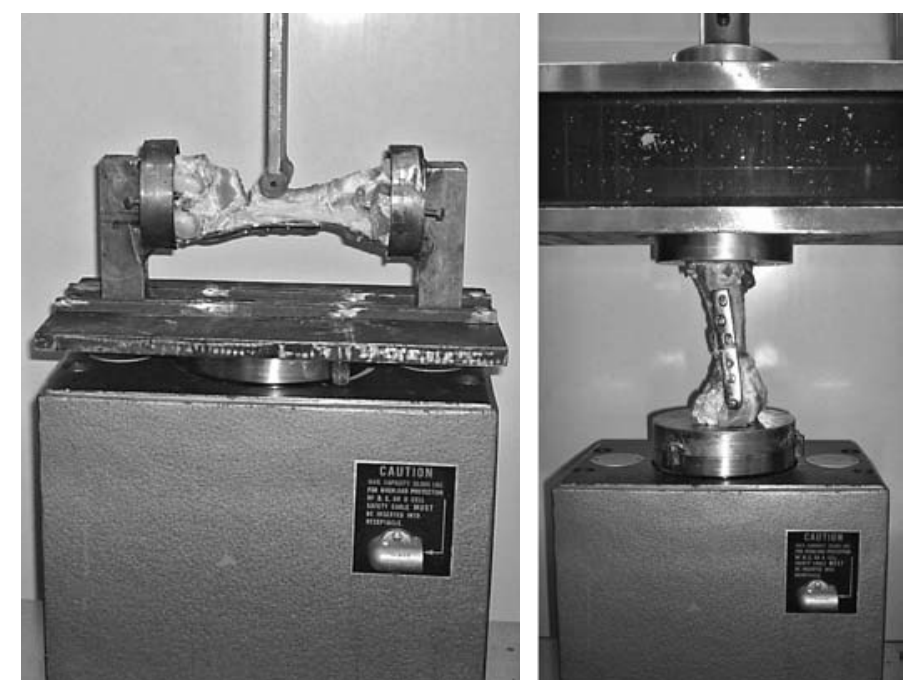

Figura 3 - Ensaio em flexão (à esquerda) e em compressão (à direita)

Após o posicionamento adequado, as peças foram submetidas a cargas que eram aumentadas progressivamente com velocidade de um centímetro por minuto ( $\mathrm{cm} / \mathrm{min})$, mensuradas por meio de gráficos analógicos medidos em quilograma-força (kgf), até o ponto de falha mecânica, que foi definida como uma alteração da forma no gráfico de força versus falha mecânica. As falhas observadas durante os ensaios ocorreram no osso (fratura) ou nos parafusos proximais das placas (soltura) e foram devidamente anotadas e analisadas estatisticamente.

\section{Metodologia estatística}

Todas as variáveis foram submetidas à análise descritiva.

Na comparação das médias das forças a que as placas foram submetidas foi utilizado o teste $t$ de Student para amostras independentes; na verificação da normalidade dos dados, o teste de Anderson-Darling e, na suposição de igualdade de variância, o teste de Levene. O teste do qui-quadrado de Pearson foi utilizado na comparação das proporções de falha no osso e no parafuso para cada tipo de placa. O nível de significância foi igual a 5\%.

\section{RESULTADOS}

A força média de flexão para a placa DCS foi de 89kgf (DP = 22), mínimo de 40kgf e máximo de 125kgf. Para a placa-lâmina a média dessa força foi de 109kgf (DP = 41), mínimo de 55kgf e máximo de 185kgf (Tabela 1). 
Tabela 1 - Distribuição das forças de flexão e compressão por tipo de placa

\begin{tabular}{l|c|c|c|c|c|c}
\hline & $\mathbf{N}$ & $\begin{array}{c}\text { Média } \\
(\mathbf{k g f})\end{array}$ & $\begin{array}{c}\text { Desvio } \\
\text { padrão }\end{array}$ & $\begin{array}{c}\text { Min. } \\
(\mathbf{k g f})\end{array}$ & $\begin{array}{c}\text { Mediana } \\
(\mathbf{k g f})\end{array}$ & $\begin{array}{c}\text { Max. } \\
(\mathbf{k g f})\end{array}$ \\
\hline Flexão \\
\hline DCS & 15 & 89 & 22 & 40 & 95 & 125 \\
\hline Lâmina & 20 & 109 & 41 & 55 & 103 & 185 \\
\hline Compressão \\
\hline DCS & 15 & 301 & 220 & 75 & 250 & 700 \\
\hline Lâmina & 15 & 276 & 97 & 165 & 250 & 505 \\
\hline
\end{tabular}

Os boxplots (Figura 4) apresentam as distribuições das forças de flexão e compressão por tipo de placa. A distribuição da força de flexão foi bem mais homogênea no DCS do que na placa-lâmina (caixa menor que indica dados mais homogêneos na amostra). Já no caso da força de compressão, observa-se que a placa-lâmina tem distribuição mais homogênea do que o DCS.
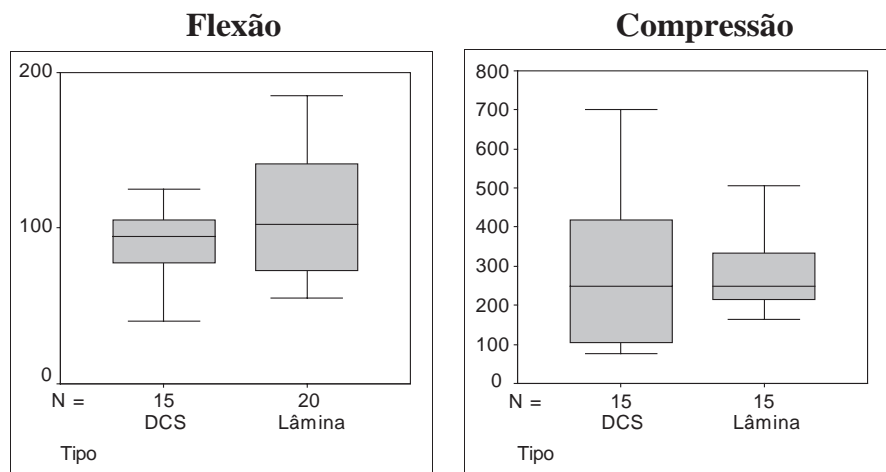

Figura 4 - Boxplots das forças de flexão e compressão em função das placas

As variáveis força de flexão e compressão em função do tipo de placa não violaram a suposição de normalidade, segundo o teste de Anderson-Darling. A suposição de igualdade de variâncias não foi assumida, segundo o teste de Levene.

Na Tabela 2 é apresentado o teste para a comparação das médias da força de flexão e compressão em função do tipo de placa. Não foi detectada diferença estatisticamente significante na média das forças de flexão $(\mathrm{t}=-1,904 ; \mathrm{p}=0,066)$ e compressão $(\mathrm{t}=0,398$; $\mathrm{p}=0,694)$ por tipo de placa, ou seja, as duas placas são iguais com relação às forças de flexão e compressão.

Para verificar se as proporções de tipos de falhas foram diferentes por tipo de placa, foi realizado o teste do qui-quadrado de Pearson. Nos testes em que se utilizou a força de flexão foi observado que a montagem com o DCS, visualmente, falhou mais no osso, no local da osteotomia, enquanto que a montagem com placa-lâmina
Tabela 2 - Teste $t$ de Student (amostras independentes) para a comparação de médias

\begin{tabular}{c|c|c}
\hline \multirow{2}{*}{} & \multicolumn{2}{|c}{ Teste $\boldsymbol{t}$ para igualdade de médias* } \\
\cline { 2 - 3 } & $\mathbf{T}$ & Valor $\mathbf{p}$ \\
\hline Flexão & $-1,904$ & 0,066 \\
\hline Compressão & 0,398 & 0,694 \\
\hline
\end{tabular}

*Igualdade de variâncias não assumida

falhou mais na região metadiafisária, no local de fixação dos parafusos. Essa diferença não foi estatisticamente significativa $\left(\chi^{2}=2,37 ; p=0,123\right)$, concluindo-se que as duas placas comportam-se de maneira semelhante em relação aos tipos de falha (Tabela 3).

Tabela 3 - Teste do qui-quadrado de Pearson para comparação de proporções de falhas por tipo (osso, parafuso) no teste de força de flexão

\begin{tabular}{c|c|c|c|c|c|c|c}
\hline Flexão & DCS & Lâmina & Total & DCS & Lâmina & & \\
\hline OSSO & 10 & 8 & 18 & $67 \%$ & $40 \%$ & $\mathbf{X}^{2}$ & 2,37 \\
\hline PARAFUSO & 5 & 12 & 17 & $33 \%$ & $60 \%$ & Valor $\mathbf{p}$ & 0,123 \\
\hline Total & 15 & 20 & 35 & & & OR & $3,00(0,61 ; 15,61)$ \\
\hline Compressão & DCS & Lâmina & Total & DCS & Lâmina & & \\
\hline OSSO & 7 & 9 & 16 & $47 \%$ & $60 \%$ & $\mathbf{X}^{2}$ & 0,52 \\
\hline PARAFUSO & 8 & 6 & 14 & $53 \%$ & $40 \%$ & Valor $\mathbf{p}$ & 0,472 \\
\hline Total & 15 & 15 & 30 & & & OR & $0,58(0,11 ; 3,13)$ \\
\hline
\end{tabular}

Nos testes em que se utilizou a força de compressão, pôde-se observar visualmente que a montagem com o DCS falhou mais na região metadiafisária, no local de fixação dos parafusos, enquanto que a montagem com placa-lâmina falhou mais no osso, na região da osteotomia. Essa diferença não foi estatisticamente significante $\left(\chi^{2}=0,52 ; p=0,472\right)$ (Tabela 3).

\section{DISCUSSÃO}

Apesar de a evolução do tratamento das fraturas supracondilianas do fêmur tender aos princípios de fixação interna rígida, possibilitando a reabilitação precoce ${ }^{(9)}$, não existe consenso sobre o material de fixação ideal ${ }^{(10-12)}$.

Firoozbakhsh et $a^{(6)}$ publicaram em 1995 um trabalho comparando haste intramedular com placa angulada a 95 graus. Utilizaram fêmures sintéticos com realização de defeito medial (osteotomia), que foram fixados com haste intramedular ou placa-lâmina a 95 graus. Os resultados mostraram que a placa apresentou maior rigidez em forças valgizantes, torção e flexão lateral. Já a diferença das forças compressivas em varo e flexão medial não foi estatisticamente significativa. 
Koval et $a l^{(7)}$ publicaram em 1996 trabalho semelhante ao de Firoozbakhsh et $a^{(6)}$; concluíram que a placa de 95 graus apresenta maior rigidez do que as hastes intramedulares tanto nas fraturas transversas quanto nas osteotomias do fêmur distal com defeito medial.

David et $a l^{(5)}$, em estudo de 1997, compararam haste intramedular com DCS em diversos padrões de fratura do fêmur distal. Para isso utilizaram fêmures de cadáver humano com diferentes padrões de osteotomias, produzindo, desse modo, diferentes fraturas que, baseadas na classificação do grupo $\mathrm{AO} / \mathrm{ASIF}^{(8)}$, variavam de $33^{\mathrm{a}}$, aumentando progressivamente a gravidade chegando até os tipos 33C. Os resultados mostraram não haver diferença entre haste intramedular e DCS de acordo com o tipo de fratura e, consequentemente, concluíram que a escolha do material de osteossíntese não deve ser baseada no padrão da fratura. Recomendaram ainda nesse estudo que, ao optar pelo DCS, este deverá ser de 12 furos em uma configuração mais dispersa dos parafusos por apresentar maior rigidez. Já optando pela haste intramedular, configuração mais agrupada dos parafusos de travamento é a de escolha.

Uma das desvantagens do uso do DCS é a perda óssea durante a preparação do furo para o parafuso dinâmico $^{(2)}$. Esse dado não foi confirmado pelo presente estudo, pois não foi observada diferença entre os dois tipos de implante quanto ao tipo de falha, se óssea ou no implante ( $\mathrm{p}=0,123$ no teste de flexão e $\mathrm{p}=0,472$ no teste de compressão).

Em ensaio biomecânico com DCS e placa-lâmina 95º Jaakkola et al ${ }^{(13)}$ concluíram que o DCS apresenta maior rigidez em compressão axial e média de carga máxima maior do que a da placa-lâmina, o que vem estar de acordo com os resultados deste trabalho, porém sem significância estatística $(p=0,694)$. Já no ensaio

\section{REFERÊNCIAS}

1. Rockwood CA, Green DP, Bucholz RW, Heckman JD, editors. Rockwood and Green's fractures in adults. 6th ed. Philadelphia: Lippincott Williams \& Wilkins; 2006.

2. Schatzker J. Fractures of the distal femur revisited. Clin Orthop Relat Res. 1998;(347):43-56.

3. Albert MJ. Supracondylar fractures of the femur. J Am Acad Orthop Surg. 1997;5(3): 163-71.

4. Ito K, Grass R, Zwipp H. Internal fixation of supracondylar femoral fractures: comparative biomechanical performance of the 95-degree blade plate and two retrograde nails. J Orthop Trauma. 1998;12(4):259-66.

5. David SM, Harrow ME, Peindl RD, Frick SL, Kellan JF. Comparative biomechanical analysis of supracondylar femur fracture fixation: locked intramedulary nail versus 95-degree angled plate. J Orthop Trauma. 1997;11(5):344-50.

6. Firoozbakhsh K, Behzadi K, DeCoster TA, Moneim MS, Naraghi FF. Mechanics of retrograde nail versus plate fixation for supracondylar femur fractures. J Orthop Trauma. 1995;9(2):152-7

7. Koval KJ, Kummer FJ, Bharam S, Chen D, Halder S. Distal femoral fixation: a laboratory comparison of the $95^{\circ}$ plate, antegrade and retrograde inserted reamed intramedullary nails. J Orthop Trauma. 1996;10(6):378-82. em flexão os resultados foram semelhantes, porém, em nosso estudo, a placa-lâmina mostrou média de carga superior à do DCS, apesar de não ter sido estatisticamente significativa ( $p=0,066)$; essa tendência poderia ser comprovada estatisticamente aumentado-se a amostra.

Harder et al $^{(14)}$ não observaram diferença na comparação biomecânica entre a placa-lâmina e o DCS no tratamento de fraturas supracondilianas do fêmur instáveis. Os autores sugerem, no entanto, como implante de escolha, o DCS, pela facilidade técnica, principalmente se o fragmento distal for de pelo menos $4 \mathrm{~cm}$ e para cirurgiões menos experientes. Esse resultado se assemelha ao do presente estudo quanto à equivalência biomecânica das placas, não se observando, porém. um implante que pudesse ser considerado de escolha, apesar da maior dificuldade técnica observada na introdução da placa-lâmina.

\section{CONCLUSÃO}

Concluindo, não houve diferença estatisticamente significante em relação à resistência às cargas em flexão e compressão, e ao tipo de falha, se óssea (fratura) ou se do material (soltura ou quebra do implante) entre a placalâmina e o DCS. No entanto, há um indicativo $(\mathrm{p}=0,066)$ de que a placa-lâmina possa apresentar rigidez maior em flexão do que o DCS. Poderíamos confirmar essa tendência se aumentássemos a amostra do grupo de placa-lâmina no ensaio em flexão. Neste estudo testamos a rigidez do conjunto placa-osso medida em kgf e não a fadiga do material de osteossíntese. Há que se ressaltar a escassez de estudos comparando as placas em questão; no entanto, os nossos achados são compatíveis com os da literatura existente em termos de equivalência entre as placas DCS e lâmina para o tratamento das fraturas supracondilianas extra-articulares do fêmur em modelos experimentais.

8. Müller ME, Allgower M, Schneider R. Manual of internal fixation. New York: SpringerVerlag; 1979.

9. Silisk J M, Mahring M, Hofer P. Supracondylar-intercondylar fractures of the femur treatment by internal fixation. J Bone Joint Surg Am. 1989;71(1):95-104.

10. Arneson TJ, Meltron LJ 3rd, Lewallen DG, O'Fallon WM. Epidemiology of diaphyseal and distal femoral fractures in Rochester, Minnesota, 1965-1984. Clin Orthop Relat Res. 1988;(234):188-94.

11. Koval KJ, Hoehl JJ, Kummer FJ, Simon, JA. Distal femoral fixation: a biomechanical comparison of the standard condylar buttress plate, a locked buttress plate, and the 95-degree blade plate. J Orthop Trauma. 1997;11(7):521-524.

12. Sanders R, Swiontkowski M, Rosen H, Helfet D. Double-plating of comminuted, unstable fractures of the distal part of the femur. J Bone Joint Surg Am. 1991;73(3):341-6.

13. Jaakkola JI, Lundy DW, Moore T, Jones B, Ganey TM, Hutton WC. Supraconylar femur fracture fixation: mechanical comparison of the $95^{\circ}$ condylar plate and screw versus $95^{\circ}$ angled blade plate. Acta Orthop Scand. 2002;73(1):72-6.

14. Harder Y, Martinet O, Barraud G-E, Cordey J, Regazzoni P. The mechanics of internal fixation of fractures of the distal femur: a comparison of the condylar screw (DCS) with the condylar plate (CP). Injury. 1999;30(Suppl 1):A31-9. 